\title{
Inhibition of CDH17 gene expression via RNA interference reduces proliferation and apoptosis of human MKN28 gastric cancer cells
}

\author{
RUI LI ${ }^{1}$, HONG-QIANG YANG ${ }^{2}$, HAI-LIN XI ${ }^{2}$, SU FENG $^{2}$ and RUI-HAO QIN ${ }^{2}$ \\ ${ }^{1}$ Department of Central Laboratory, ${ }^{2}$ Department of General Surgery, Xuzhou Central Hospital, Xuzhou Clinical School \\ of Xuzhou Medical College, Affiliated Hospital of Southeast University, Xuzhou, Jiangsu 221009, P.R. China
}

Received September 26, 2016; Accepted October 24, 2016

DOI: $10.3892 /$ ijo.2016.3783

\begin{abstract}
Gastric cancer is the fourth most common type of cancer and the second cause of cancer-related mortalities worldwide despite the use of multimodal therapy. Cadherins are transmembrane glycoproteins that are involved in tumorigenesis. $\mathrm{CDH} 17$ has been found to be over-expressed in gastric cancer and its overexpression was associated with lymph node metastasis and tumor-node-metastasis stage of the patients, yet the exact role and molecular mechanism of CDH17 in gastric cancer have not been determined. Using a lentiviral system as a delivery mediator of RNA interference, we found that inhibition of CDH17 can lead to reduce proliferation and increase apoptosis of gastric cancer cell line MKN28 in vitro and significantly diminish their tumorigenicity in vivo. Our results of the present study suggest that $\mathrm{CDH} 17$ may be a promising candidate for the therapeutic targeting of gastric cancer.
\end{abstract}

\section{Introduction}

Gastric cancer is the fourth most common type of cancer and the second cause of cancer-related mortalities worldwide (1). Many Asian countries, including China, Japan and Korea, have extremely high rates of gastric cancer (2). Despite the use of multimodal therapy, there are no effective methods for the treatment of gastric cancer. Therefore, identifying the new therapeutic targets is needed.

Cadherins are transmembrane glycoproteins mediating $\mathrm{Ca}^{2+}$-dependent adhesion of adjacent cells and have strong implications in tumorigenesis (3). Liver-intestine cadherin (CDH17), also known as LI-cadherin, is a new member of

Correspondence to: Dr Hai-lin Xi, Department of General Surgery, Xuzhou Central Hospital, Xuzhou Clinical School of Xuzhou Medical College, Affiliated Hospital of Southeast University, 199 South Jiefang Road, Xuzhou, Jiangsu 221009, P.R. China E-mail: xhl-430@163.com

Key words: gastric cancer, RNA interference, $\mathrm{CDH} 17$, proliferation, apoptosis the cadherin superfamily. It is distinguished from classical cadherins by its functional features and unique structure, as it contains seven rather than five molecular domains $(3,4)$. Previous studies revealed that CDH17 is highly expressed in many types of cancer, such as gastric, liver, intestinal and pancreatic (5). However, the expression of $\mathrm{CDH} 17$ is negligible in the epithelium of the healthy human stomach. Ito et al demonstrated that $\mathrm{CDH} 17$ was one of the most upregulated genes in gastric carcinomas and its expression of CDH17 was correlated with poor prognosis (6). CDH17 is also a useful immunohistochemical marker for the diagnosis of gastric metaplasia and neoplasia (5). Despite these significant clinical findings, the functions of CDH17 in human cancers are unclear and controversial.

In the present study, we used a lentiviral system as a delivery mediator of RNA interference (RNAi) and established a stable CDH17-silencing gastric cell line, and evaluated the effects of CDH17 suppression on the migration, invasion, proliferation and apoptosis of gastric cancer cells in vitro as well as tumor growth in vivo.

\section{Materials and methods}

Patients and tissue specimens. Paraffin-embedded tumors and their adjacent non-cancerous tissues specimens were collected from a series of 79 patients with gastric carcinoma who had undergone surgical resection between 1 January, 2007 and 31 December, 2007 at the First Affiliated Hospital of Medicine School, Shihezi University, Xinjiang, China. There were 56 men (71\%) and 23 women (29\%). The mean age was 60.1 years (range, 27-80 years). The specimens were classified by their tumor-node-metastasis (TNM) stage (UICC, 2010). Detailed patient information such as gender, age, depth of invasion and lymphatic invasion is listed in Table I.

Immunohistochemistry (IHC). Paraffin-embedded 4- $\mu \mathrm{m}$ tissue sections were stained for CDH17 using anti-LI cadherin antibody (ab109220; Abcam, Cambridge, UK). In brief, paraffin-embedded tissues were deparaffinized through graded xylene, treated with $3 \%$ hydrogen peroxide in methanol for $10 \mathrm{~min}$, and immersed in $10 \mathrm{mmol} / 1$ citrate buffer ( $\mathrm{pH} \mathrm{6.0)}$. The specimens were incubated with $10 \%$ normal goat serum 
Table I. Association between CDH17 expression and the clinicopathological characteristics of gastric carcinoma.

CDH17 expression

Parameters

Negative $(n=30)$

Positive $(n=49)$

P-value

Age (mean \pm SD, years)

$59.4 \pm 12.0$

$60.6 \pm 13.7$

Gender

Male

19

Female

11

Histologic type

Well-differentiated

1

Moderately

13

Poorly differentiated

16

Depth of invasion

T1

T2

5

24

1

T3

0

Lymph node metastasis

Absent
Present
TNM stage
0+I+II
III+IV

17

13

19

11

Distant metastasis

M0

29

1
37

12

$0.020^{\mathrm{a}}$

$0.022^{\mathrm{a}}$

0.248

4

14

31

6

30

6

7

15

34

$0.021^{\mathrm{a}}$

18

31

43

6

${ }^{\mathrm{a}} \mathrm{P}<0.05$, statistical significance.

(catalog no.: SA1022, Boster, Wuhan, China), followed by the primary rabbit polyclonal antibody against $\mathrm{CDH} 17$ (diluted in 1:400; catalog no.: ab3163-1,Abcam,Cambridge, MA, USA) at $4^{\circ} \mathrm{C}$ overnight and the biotinylated goat anti-rabbit IgG secondary antibody (catalog no.: SA1022, Boster, Wuhan, China) at $37^{\circ} \mathrm{C}$ for $30 \mathrm{~min}$. After washing, 3,3'-diaminobenzidine tetrahydrochloride solution was used to visualise the staining. The stained slides were evaluated by standard light microscopy (Eclipse 80i; Nikon, Tokyo, Japan).

Cell culture, lentivirus production and transduction. Human MKN28 gastric cancer cells were cultured in RPMI-1640 with $10 \%$ fetal bovine serum, $100 \mathrm{U} / \mathrm{ml}$ penicillin and $100 \mu \mathrm{g} / \mathrm{ml}$ streptomycin in a humidified incubator supplemented with $5 \% \mathrm{CO}_{2}$ at $37^{\circ} \mathrm{C}$. The lentiviral RNAi expression vector with specific miRNA against $\mathrm{CDH} 17$ (lenti-shCDH17) was constructed as previously described (7-9). The lentiviral vector was used to transduce MKN28 cells and the stably transfected cell line termed lenti-shCDH17, in which CDH17 had been knocked down by RNAi. The other two groups comprised MKN28 cells received no treatment and the empty vector-transfected control cells (lenti-shCDH17-neg).

Immunofluorescent staining. The expression of CDH17 was determined by IF using anti-LI cadherin antibody (ab69602;
Abcam). Briefly, MKN28 cell cultures, grown in 6-well plates, on coverslips, were fixed with $4 \%$ paraformaldehyde for $10 \mathrm{~min}$. Fixed cells were permeabilized in $0.1 \%$ Triton X-100 for $5 \mathrm{~min}$. Rabbit anti-LI cadherin sera at a dilution of 1:500 were applied to these wells and incubated at $37^{\circ} \mathrm{C}$ for $2 \mathrm{~h}$. The cells were washed three times with PBS for 5 min each time. Goat anti-rabbit IgG conjugated with cy3 (Sigma) at a dilution of 1:50 was added and incubated for $40 \mathrm{~min}$ at $37^{\circ} \mathrm{C}$. After being washed three times and stained with DAPI (diluted in 1:2,000) for $5 \mathrm{~min}$, the glass slides were mounted in glycerol with coverslips and the cells were observed under an inverted fluorescence microscope (Olympus, Tokyo, Japan).

Western blotting. The MKN28 cells of the different groups were harvested, washed with phosphate-buffered saline [PBS, pH 7.4) and treated with trypsin. All the cells were lysed in RIPA buffer [50 mM Tris-HCl, pH 7.4, $150 \mathrm{mM}$ $\mathrm{NaCl}, 1 \% \mathrm{NP}-40,0.5 \%$ sodium deoxycholate, $0.1 \%$ sodium dodecyl sulfate (SDS), $1 \mathrm{mM}$ EDTA, $30 \mu \mathrm{g} / \mathrm{ml}$ aprotinin, $50 \mu \mathrm{g} / \mathrm{ml}$ leupeptin and $1 \mathrm{mM}$ PMSF]. Protein was loaded at a concentration of $30 \mu \mathrm{g}$ per lane and separated on a $10 \%$ SDS-polyacrylamide gel electrophoresis (PAGE). After electrophoresis, the protein was transferred onto a polyvinyl difluoride membrane, incubated with $5 \%$ non-fat milk for $1 \mathrm{~h}$ and then incubated overnight at $4{ }^{\circ} \mathrm{C}$ with anti-CDH17 antibody 
(1:1,000; Abcam). Horseradish peroxidase-conjugated IgG was used as the secondary antibody. Protein was visualized by the enhanced chemiluminescence kit.

Quantitative polymerase chain reaction ( $q P C R)$. We extracted total RNA using TRIzol reagent (Invitrogen, Hong Kong, China), and single-stranded cDNA performed with the cDNA synthesis kit (Takara Bio, Inc., Otsu, Japan) according to standard protocols. We performed qPCR using SYBR ${ }^{\circledR}$ Premix $\mathrm{Ex} \mathrm{Taq}^{\mathrm{TM}}$ II (Takara Bio, Inc.). The reactions were carried out in a $10-\mu 1$ volume reaction system. The PCR conditions consisted of 45 cycles, with $5 \mathrm{sec}$ denaturation at $95^{\circ} \mathrm{C}, 30 \mathrm{sec}$ annealing at $60^{\circ} \mathrm{C}$ and $1 \mathrm{~min}$ at $72^{\circ} \mathrm{C}$. The primers used were: CDH17, forward: 5'-GCCAATCCTCCTGCTGTG-3' and reverse: 5'-GCAACCTGGAGATTGTGAGT-3'; GAPDH, forward: 5'-TGACTTCAACAGCGACACCCA-3', and reverse: 5'-CACCCTGTTGCTGTAGCCAAA-3'. The reactions were performed in triplicate. The relative expression level of $\mathrm{CDH} 17$ gene was calculated based on the method of $2^{-\Delta \Delta \mathrm{Cq}}$.

In vitro migration and invasion assay. The inhibitory effect of RNAi on MKN28 migration and invasion in vitro was demonstrated in transwell chambers $(8.0 \mu \mathrm{m}$ pore size; Corning, NY, USA) according to the manufacturer's instructions. First, transwell chambers were placed onto 24 -well plates. The cells were then resuspended in serum-free medium $\left(1 \times 10^{6}\right.$ cells $\left./ \mathrm{ml}\right)$, and $100 \mu \mathrm{l}$ of this suspension was added to the upper chambers. The lower chambers were filled with $300 \mu \mathrm{l}$ of RPMI-1640 medium containing $15 \%$ fetal bovine serum. Transwell invasion chambers were coated on the upper surface with $50 \mu \mathrm{l}$

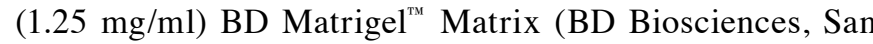
Diego, CA, USA). Following 24-h incubation, the cells on the lower surface of the filters were fixed in methanol, stained with $0.25 \%$ crystal violet for $15 \mathrm{~min}$ and counted in five random fields at a magnification of $\times 200$.

Flow cytometry for the determination of MKN28 cell apoptosis. An Annexin V/propidium iodide (PI) apoptosis detection kit I (BD Biosciences) was used to measure apoptosis. The cells from each group were collected, washed twice with ice-cold PBS and re-suspended in $200 \mu \mathrm{l}$ binding buffer containing $10 \mu \mathrm{l}$ Annexin V and $10 \mu \mathrm{l}$ PI. Fluorescence intensity was measured by flow cytometry (BD Biosciences). All the samples were assayed in triplicate.

Measurement of cell proliferation by the cell counting kit- 8 (CCK-8) assay and colony formation assay. To observe the effect of the $\mathrm{CDH} 17$ gene expression on cell proliferation in vitro, CCK-8 was employed to draw cell growth curves. The cells from each group were plated onto 96-well cell culture plates at a density of $2 \times 10^{4}$ cells/well in $100 \mu 1$ of culture medium containing $10 \%$ fetal bovine serum and grown overnight. Each group had 5 replicates holes. At 24, 48, 72, 96 and 120 h, CCK-8 solution was added to each well and the plate was placed in a $\mathrm{CO}_{2}$ incubator for $4 \mathrm{~h}$. The plate was then placed in a microplate reader (Bio Rad, Hercules, USA and the O.D. at $450 \mathrm{~nm}$ was read. A colony formation assay was performed to analyze anchorage-dependent growth. The cells were plated on a 6 -well plate at $1 \times 10^{3}$ cells/well. Colonies were stained with $0.1 \%$ crystal violet and counted on the 14 th day after seeding. Colonies containing $\geq 50$ cells were counted. The experiments were carried out in triplicate.

In vivo mouse models of gastric cancer and tumorigenicity assay. Animal experiments were approved by the Animal Experimental Ethics Committee of the Huazhong University. Gastric cancer xenografts were established in 5-week-old male BALB/c nude mice (Beijing HFK Bioscience Co. Ltd., Beijing, China). Briefly, the cells from the three groups were harvested by trypsinization, and washed with PBS. Single-cell suspensions (6x10 6 cells in $200 \mu \mathrm{l}$ PBS) were injected into the nude mice (six mice per group). Subcutaneous tumors were monitored every 3 days with a caliper and tumor growth curves were calculated. Tumor size was calculated using the formula: Length $\mathrm{x}$ width ${ }^{2} \mathrm{x} 0.5$. After 4 weeks, the mice were sacrificed using cervical dislocation and the xenograft tumor tissues were harvested, weighed, fixed, and embedded. The slides were incubated with the primary antibody against Ki-67 (Santa Cruz Biotechnology, Inc., Dallas, TX, USA) and caspase-3 (Santa Cruz Biotechnology, Inc.) overnight at $4^{\circ} \mathrm{C}$. Biotinylated goat anti-rabbit IgGs or goat anti-mouse IgGs were used as secondary antibodies. According to the manufacturer's protocol of the TUNEL Apoptosis Assay kit (Roche Diagnostics, Indianapolis, IN, USA), TUNEL was performed to detect apoptosis in the tumor sections. Five fields were randomly selected from each sample and 200 cells were randomly selected from every field under microscopy. The apoptotic rate was calculated as the number of total apoptotic cells/1,000) x 100\%.

Statistical analysis. The experiments were repeated three times. Statistical analyses were performed using SPSS 12.0 software (SPSS Inc., Chicago, IL, USA). The results of the present study were presented as mean \pm SD. Statistically significant differences between groups for each assay were analyzed by one-way analysis of variance (ANOVA). The association between $\mathrm{CDH} 17$ protein expression and the clinicopathological characteristics was assessed by the $\chi^{2}$ test. $\mathrm{P}<0.05$ was considered to indicate a statistically significant difference.

\section{Results}

CDH17 overexpression in gastric carcinoma. CDH17 immunoreactivity was detected in 49/79 (62.1\%) gastric cancer tissues. The expression of $\mathrm{CDH} 17$ was localized in the cell membrane and cytoplasm at various levels in gastric carcinoma tissues. The high expression of CDH17 protein was observed in the cell membrane of epithelial cells with intestinal metaplasia. CDH17 was detected in the tumors with well-differentiated and moderately gastric cancer tissues. CDH17 staining intensity was relatively lower in the tumors with poor differentiation. CDH17 was absent in normal gastric tissues. Representative images of IHC staining are shown in Fig. 1.

The association between $\mathrm{CDH} 17$ expression and clinicopathological parameters is summarized in Table I. Of the 79 cases, $49(62.0 \%)$ were classified as having positive $\mathrm{CDH} 17$ expression, whereas the remaining $30(38.0 \%)$ were classified as having a negative $\mathrm{CDH} 17$ expression. A positive expression 

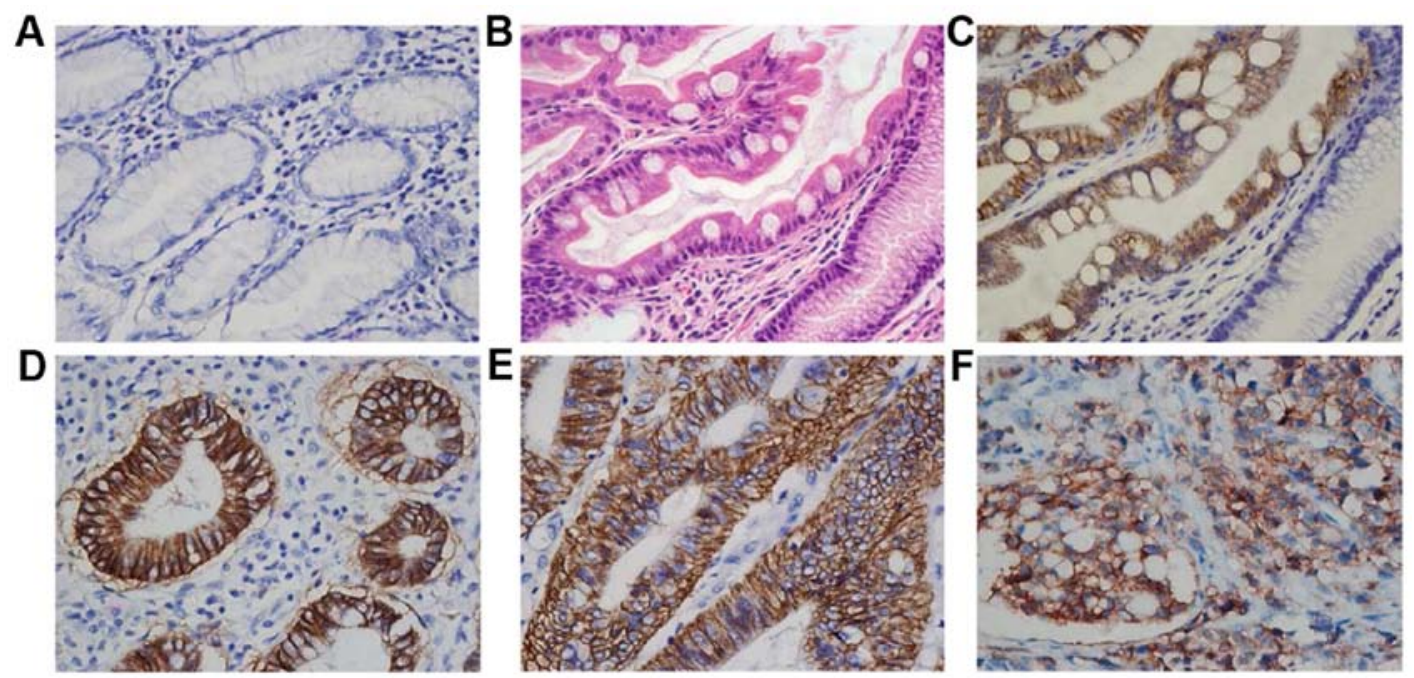

Figure 1. (A) CDH17 immunohistochemical staining was negative in normal gastric epithelium. (B) Non-neoplastic mucosa with intestinal metaplasia (H\&E staining), (C) which revealed expression of CDH17. Immunohistochemical staining with CDH17 in (D) well-, (E) moderately and (F) poorly differentiated gastric cancer tissue (original magnification, $\mathrm{x} 400$ ).

A

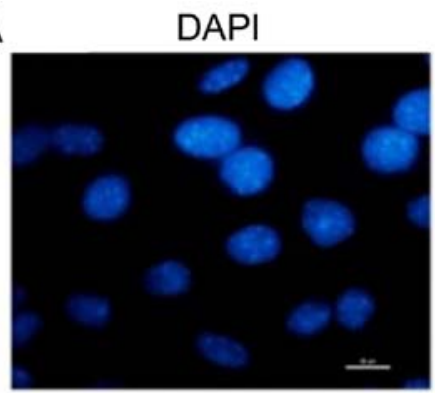

B

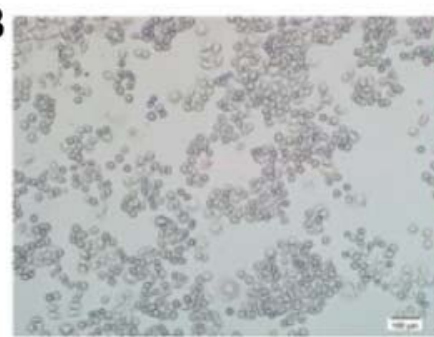

D

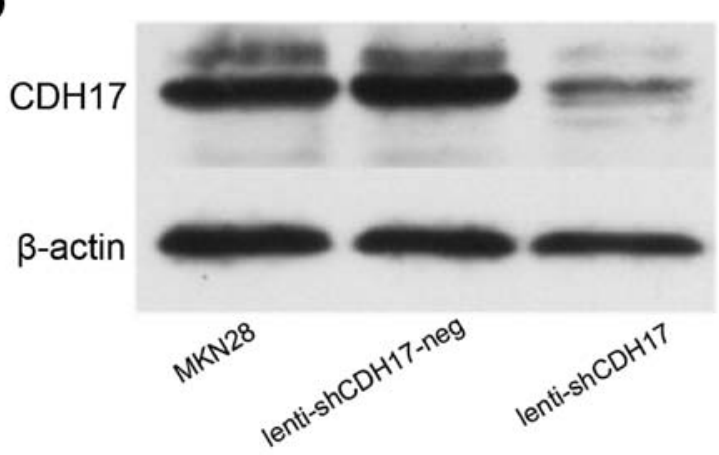

$\mathrm{CDH} 17$
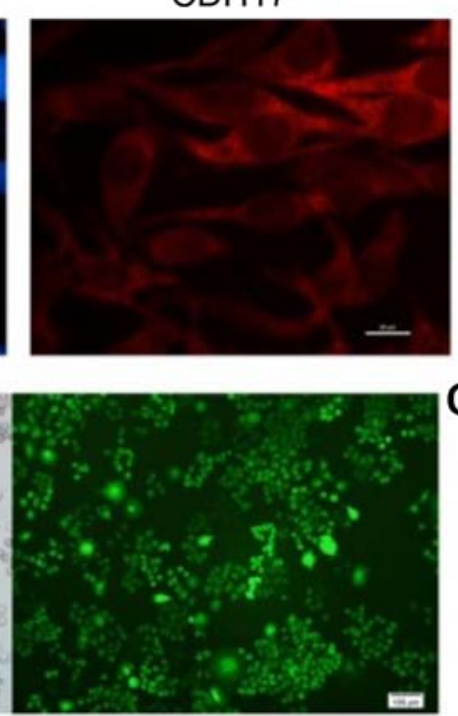

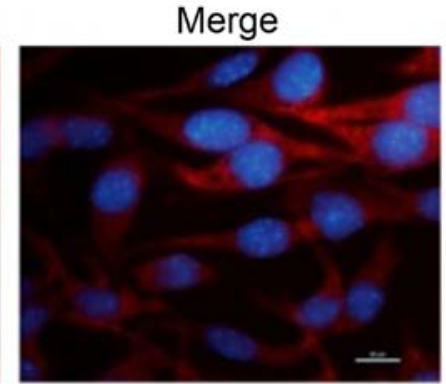

C

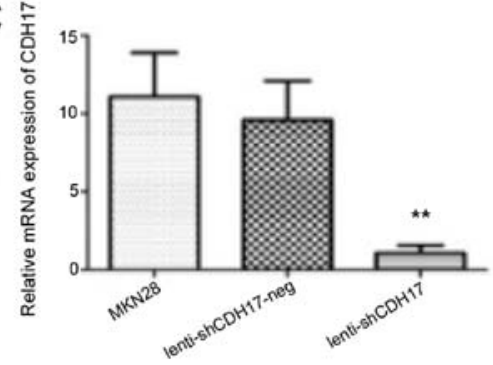

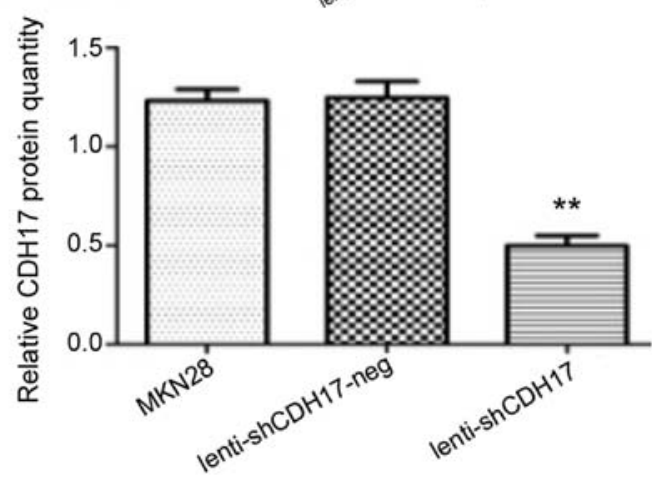

Figure 2. (A) Expression of CDH17 in MKN28 cells by immunofluorescence (magnification, x1,000). (B) Fluorescence photomicrographs of gastric cancer cells infected by lenti-shCDH17. Images were captured $72 \mathrm{~h}$ after infection. (C) Quantitative PCR analysis showed the inhibition of CDH17 at the mRNA level in lenti-shCDH17 cells. (D) Decreased protein level of CDH17 as shown by western blotting. (Each bar is the mean \pm SD. ${ }^{* *} \mathrm{P}<0.01$ ).

of $\mathrm{CDH} 17$ was associated with the depth of invasion $(\mathrm{P}=0.020)$. There was a significant relationship between lymph node metastasis and the positive expression of $\mathrm{CDH} 17(\mathrm{P}=0.022)$.
The incidence of cases with CDH17-positive expression was significantly higher in stage III-IV than in stages 0 -II $(\mathrm{P}=0.021)$. There was no significant correlation between $\mathrm{CDH} 17$ and the 

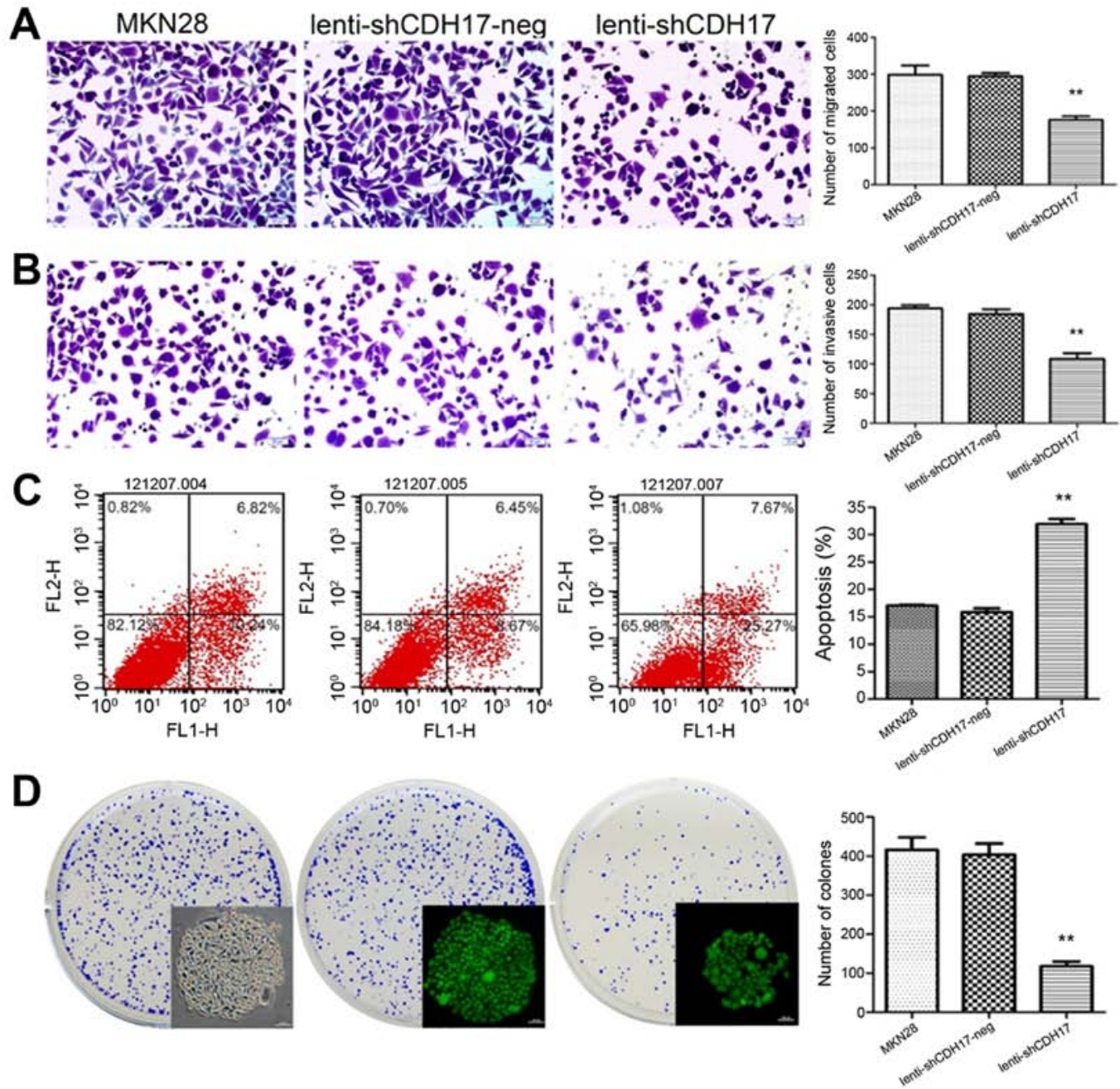

E
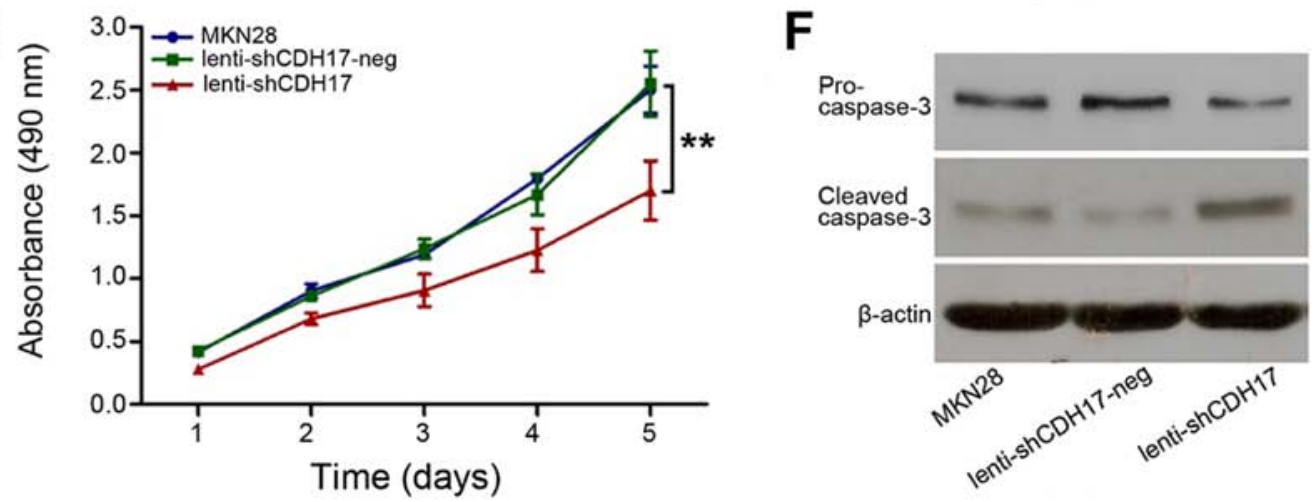

Figure 3. The effect of CDH17 downregulation on cell growth in vitro. MKN28 cells without any treatment and non-targeted RNAi vector-mediated lenti-shCDH17-neg cells were used as controls. (A) Cell migration. (B) Cell invasion. (C) Annexin V and propidium iodide double staining detected apoptosis. (D) Colony formation. (E) Cell proliferation. Knockdown of CDH17 caused a significant growth inhibition of gastric cancer cells as revealed by different assays. (F) Pro-caspase- 3 and cleaved caspase- 3 were examined by western blotting. ${ }^{*} \mathrm{P}<0.05$; ${ }^{* *} \mathrm{P}<0.01$.

other clinicopathological characteristics such as age, gender and histologic type $(\mathrm{P}>0.05)$.

Knockdown of $\mathrm{CDH} 17$ inhibits gastric cancer cell growth in vitro. In order to evaluate the effects of $\mathrm{CDH} 17$ knockdown on the $C D H 17$ gene, we selected the well-differentiated MKN28 cell line, which has a strong expression of $C D H 17$. Immunofluorescent staining showed that CDH17 was mainly present on the cell membrane and cytoplasm (Fig. 2A). As shown in Fig. 2B, lenti-shCDH17 effectively infected MKN28 cell lines. The cells were infected by lenti-CDH17 in terms of GFP expression as observed under fluorescent microscope. Subsequent to the successful infection of MKN28 cells, fluorogenic qPCR was performed to evaluate the reduction in mRNA for CDH17 in lenti-shCDH17 cells. Compared with the lenti-shCDH17-neg and untreated MKN28 cells, the mRNA expression of CDH17 was suppressed efficiently in lenti-shCDH17 cells (Fig. 2C). In addition, western blot analysis revealed a reduced expression of $\mathrm{CDH} 17$ protein in lenti-shCDH17 cells transfected with lenti-shCDH17, whereas 


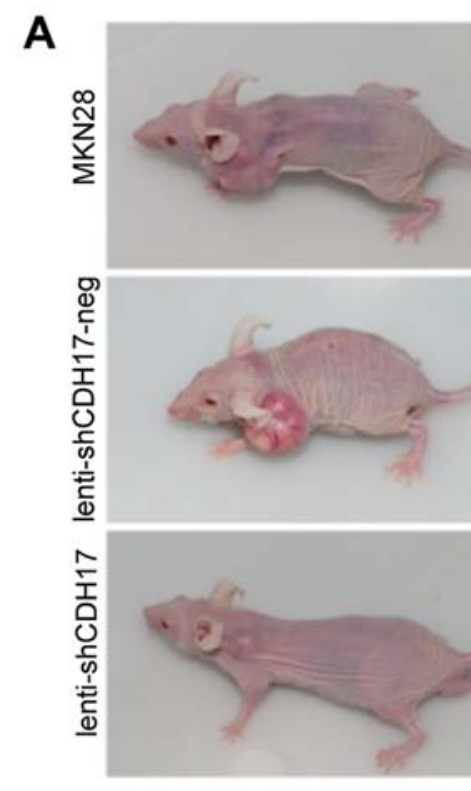

B

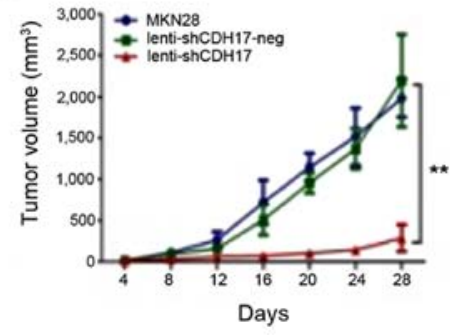

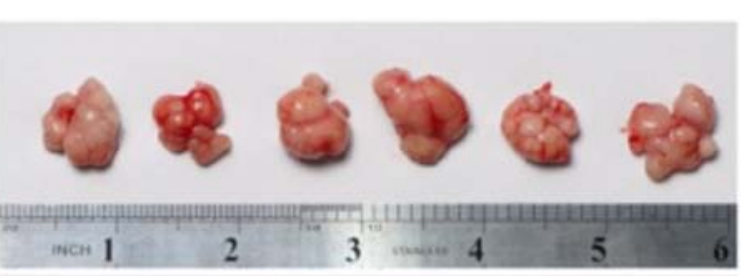
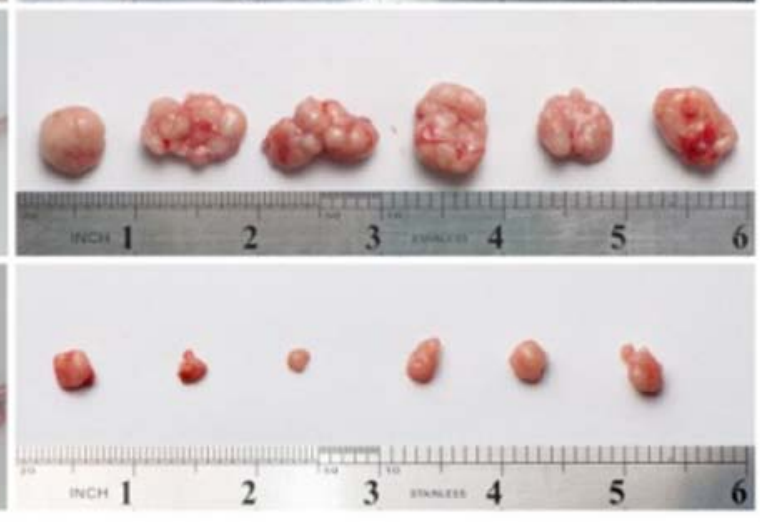

C

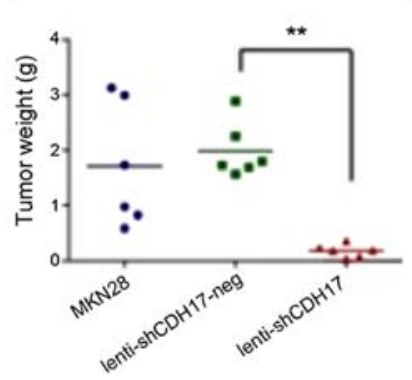

D

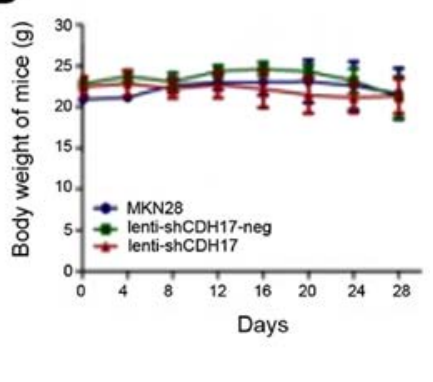

Figure 4. Knockdown of CDH17 inhibited tumor growth in a gastric cancer tumor model. (A) Images of tumors derived from MKN28, lenti-shCDH17-neg and lenti-shCDH17 cells in nude mice. (B) The changes in volume of the tumors of different groups. (C) Individual tumor weight at time of sacrifice. (D) Average body weight of nude mice measured at different time points. Data are presented as mean $\pm \mathrm{SD}$. ${ }^{* *} \mathrm{P}<0.01$.

CDH17 protein expression in lenti-shCDH17-neg cells and untreated MKN28 cells was not reduced (Fig. 2D). These results demonstrated that the expression of $\mathrm{CDH} 17$ may be downregulated specifically and effectively by lenti-shCDH17. These infected cells were used for subsequent experiments.

We investigated the tumorigenic and metastatic properties (cell migration, invasion, colony formation, apoptosis and proliferation) of the MKN28 cells transfected with lenti-shCDH17 compared with the control cells-lenti-shCDH17-neg cells and untreated MKN28 cells. Lenti-shCDH17 cells in culture showed a decreased rate of migration and invasion ability $(\mathrm{P}<0.01)$ (Figs. 3A and B). As shown in Fig. 3C, CDH17 could significantly increase the apoptotic rate of lenti-shCDH17 cells $(\mathrm{P}<0.01)$. To investigate the mechanism of apoptosis in vitro, we examined the expression of related proteins by western blot analysis. The results revealed that knockdown of CDH17 protein markedly decreased the levels of pro-caspase- 3 and increased the levels of cleaved caspase- 3 compared with the control cells (Fig. 3F). In the colony forming assay, the colony numbers of lenti-shCDH17 cells (108.67 $\pm 9.64 /$ well) were much lower than those of the control group treated with GFP-lentivirus (lenti-shCDH17-neg) cells (184.33 $\pm 8.14 /$ well) and the MKN28 cells $(193.80 \pm 5.37 /$ well $)(\mathrm{P}<0.01)$, which suggests that RNAi with specific sequence of CDH17 may significantly suppress the colony-forming ability of MKN28 cells. No significant difference was found between the lenti-shCDH17-neg and MKN28 groups ( $\mathrm{P}>0.05)$ (Fig. 3D). Empty lentiviral vector had no effect on the proliferative ability of MKN28 cells, whereas RNAi specific to MKN28 caused a marked reduction in cell proliferation $(\mathrm{P}<0.01)$ (Fig. $3 \mathrm{E})$.

Knockdown of $\mathrm{CDH} 17$ inhibits the growth of the tumor derived from MKN28 cells in vivo. Based on the data obtained from the in vitro experiments, we investigated whether the knockdown of CDH17 suppressed tumor growth and induced apoptosis in vivo. Single-cell suspensions were injected subcutaneously into the BALB/c nude mice. Four weeks after inoculation in nude mice, we found that the mean tumor volume of the lenti-shCDH17 group was $180.23 \pm 115.16 \mathrm{~mm}^{3}$, much smaller than that of the MKN28 group $\left(1708.2 \pm 1118.85 \mathrm{~mm}^{3}\right)$ and the lenti-shCDH17-neg group $\left(1988.30 \pm 500.50 \mathrm{~mm}^{3}\right)$ $(\mathrm{P}<0.01)$ (Fig. 4). Thus, these data indicate that knockdown of $\mathrm{CDH} 17$ in gastric cancer cells can inhibit the tumor formation of gastric cancer cells in vivo.

To confirm the effect of CDH17 knockdown on apoptosis in vivo, immunohistochemical analyses on the xenograft tissues were conducted. The expression of $\mathrm{CDH} 17$ was reduced in the lenti-shCDH17 group as is shown in the IF staining. In addition, the area of apoptosis in the lenti-shCDH17 group was significantly larger than the other two groups. Apoptotic 
A
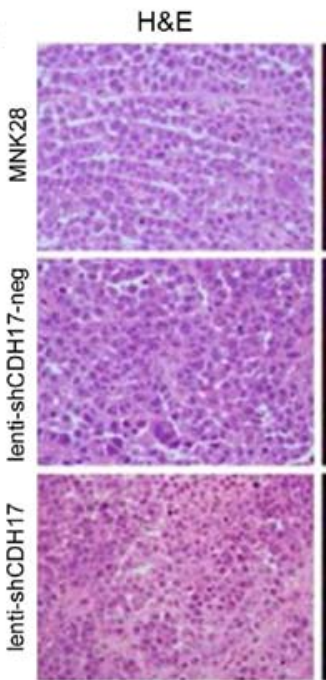
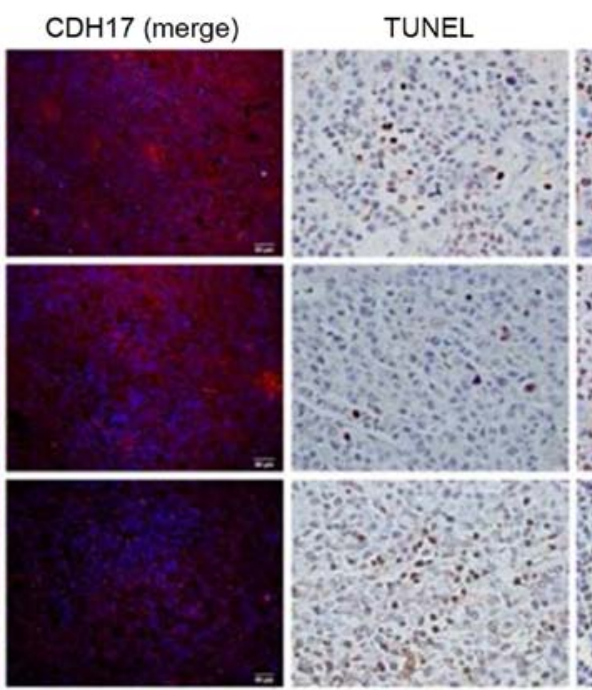

Ki-67
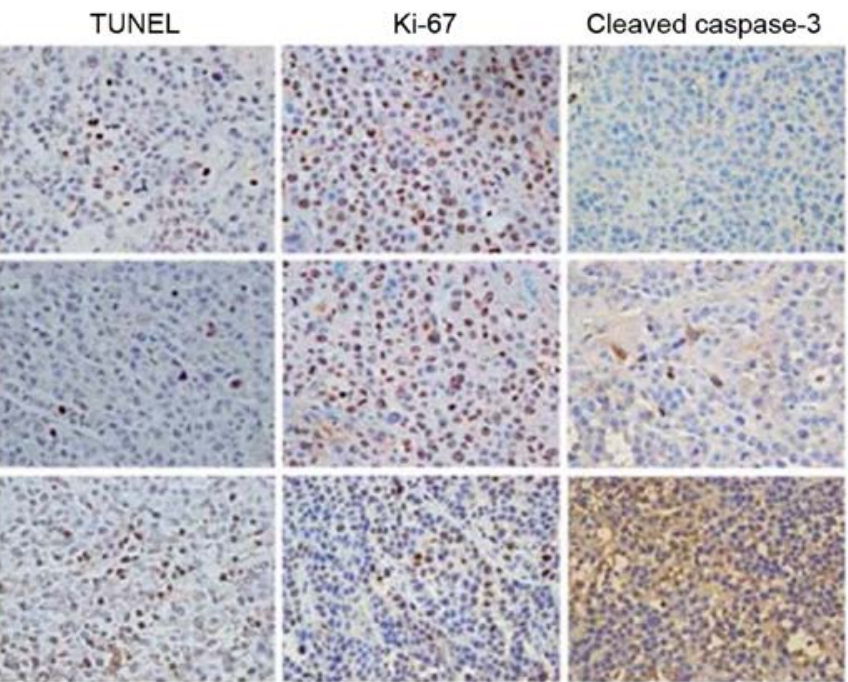

B

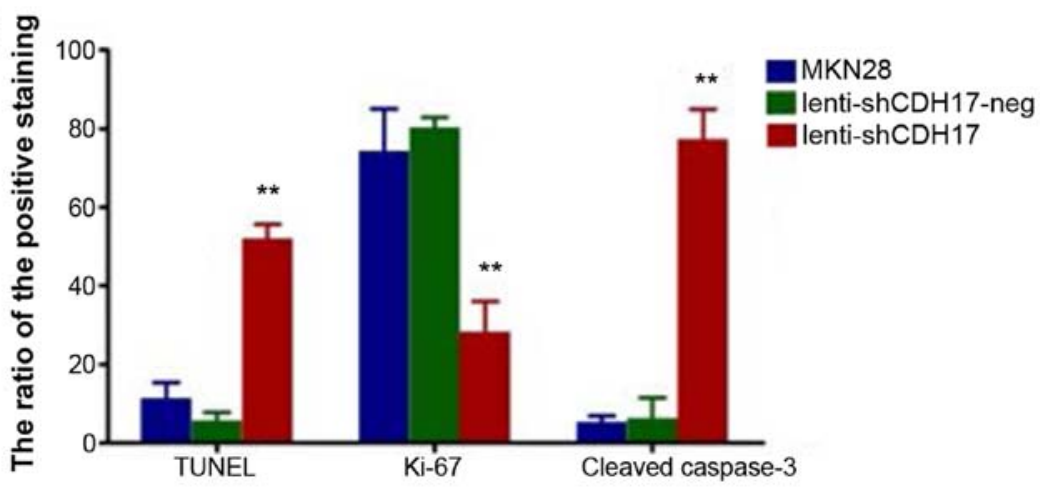

Figure 5. (A) Pathological evaluation of tumor specimens from nude mice was determined by H\&E staining (original magnification, $\mathrm{x} 400$ ). Frozen sections of xenograft tissues were used to examine the expression levels of CDH17. The reduction of CDH17 in lenti-shCDH17 cells was detected by immunofluorescent staining. All the nuclei were stained with DAPI. The expression of CDH17 is shown in red (original magnification, $\mathrm{x} 200$ ). Increased level of TUNEL and cleaved caspase-3, and decreased expression of Ki-67 antigen detected by immunohistochemistry (original magnification, $\mathrm{x} 400$ ). (B) Quantification of the ratio of positive staining in each tumor tissue region. Data are shown as mean $\pm \mathrm{SD}$. ${ }^{* *} \mathrm{P}<0.01$.

cells in xenograft sections were analyzed by TUNEL staining. We observed positive cells $(52 \pm 3.60)$ in the lenti-shCDH17 group, which was more significant than that in the MKN28 group (11.33 \pm 4.04$)$ and lenti-shCDH17-neg group (5.67 \pm 2.08$)$. The number of Ki-67-positive cells was lower in the tumor section derived from lenti-shCDH17 cells than that in lenti-shCDH17-neg or MKN28 cells $(\mathrm{P}<0.01)$. A decreased expression of $\mathrm{Ki}-67$ antigen indicated that reduced tumor growth in mice was partly due to lower proliferation caused by the knockdown of CDH17. By contrast, knockdown of CDH17 induced tumor growth and widespread staining for cleaved caspase-3, compared to others, in which the staining intensity in the lenti-shCDH17 group was significantly higher than that in the lenti-shCDH17-neg and MKN28 groups (Fig. 5).

\section{Discussion}

Previous findings have clearly demonstrated that CDH17 is linked significantly to a high incidence of tumorigenesis in the human stomach, liver, pancreas and intestine by displaying an aberrant expression in their cancerous state. CDH17 was strongly regulated by CDX2 in normal, metaplastic, and neoplastic tissues of the gastrointestinal tract (10-12). After the first report of $\mathrm{CDH} 17$ as a useful marker for the diagnosis of gastric metaplasia and neoplasia (13), there is accumulating evidence that CDH17 was expressed in $61-65 \%$ of gastric cancer tissues $(9,12,14,15)$, similar to our data here. In addition, we found that $\mathrm{CDH} 17$ was overexpressed in gastric cancer and its overexpression was associated with lymph node metastasis and TNM stage of the patients. Recent studies showed that long-term intestinal metaplasia of gastric mucosa often led to gastric adenocarcinoma (16). Furthermore, the expression of CDH17 was higher in the intestinal type than that in the diffuse type of gastric cancer (17). A high level of CDH17 tends to be correlated with advanced tumor stages and is associated with lymph node metastasis and poor prognosis $(6,18,19)$. Although it has been shown that $\mathrm{CDH} 17$ has a role in the invasion and migration of gastric cancer cells $(8,9,20)$, the molecular pathogenesis of CDH17 has not been fully clarified (21). In this report, we investigated whether the downregulation of CDH17 inhibited gastric cancer progression and attempted to elucidate its unclarified mechanisms. The powerful lentiviral RNAi expression vector with specific miRNA against $\mathrm{CDH} 17$ gene expression was used in the present study.

In the present study, we carried out transwell migration, invasion, cell proliferation and colony formation assays after CDH17 knockdown in the highly tumorigenic MKN28 cell line. Our present data show that inhibition of CDH17 by 
RNAi lead to less tumorigenesis of MKN28 cells in vitro. In addition, BALB/c-nu mice were used to investigate whether the downregulation of $\mathrm{CDH} 17$ retarted the growth of gastric cancer derived from MKN28 cells. We found that the average tumor volume and weight were significantly lower in the lenti-shCDH17 group compared with the MKN28 group and the control group treated with GFP lentivirus. The results of the present study, suggest that $\mathrm{CDH} 17$ knockdown did not eliminate the tumor completely, however, it might be more reasonable to obstruct multiple pathways simultaneously (22). In addition, $\mathrm{CDH} 17$ is not the only protease involved in the invasion and growth of gastric cancer.

Unlike gastric cancer and hepatocellular carcinoma (23), the expression of $\mathrm{CDH} 17$ is reduced in colorectal carcinoma tissues, and this low level of $\mathrm{CDH} 17$ is correlated to dedifferentiation, advanced tumor stage, tumor invasion and poor survival $(24,25)$. Taken together, CDH17 manifests unique and distinct roles in tumorigenesis originated from different organs and its molecular pathogenesis has not been fully clarified. In the present study, we investigated whether the downregulation of CDH17 induced apoptosis and inhibited the proliferation of gastric cancer cells. In vitro, CDH17 suppressed cell viability through activation of cleaved caspase- 3 and eventually induced apoptosis of MKN28 cells. In gastric cancer xenografts, we found that $\mathrm{CDH} 17$ prominently suppressed xenograft growth by inducing apoptosis, which was consistent with our study in vitro. Induction of apoptosis is considered a requirement for the inhibition of tumor growth. CDH17 can block caspase-dependent apoptosis by binding to caspase- 3 . Caspase-3 is activated in the typical apoptosis pathways (26). In addition, we observed a significant decrease in $\mathrm{Ki}-67$ in lenti-shCDH17 xenograft tissue, which indicated that reduced tumor growth was partly due to cell growth inhibition, although the underlying mechanisms need further elucidation.

In conclusion, the present study demonstrates that $\mathrm{CDH} 17$ functions as a tumor suppressor protein in gastric cancer cells in vitro and in vivo, at least in part, by inhibiting proliferation, migration and invasion via the suppression of apoptosis.

\section{Acknowledgements}

This project was partly supported by the National Natural Science Foundation of China (no. 81172294). We would like to thank Ming Yuan, La-ti Mu, Shao-xiong Niu, Yu Xi, Jia-geng He and Jun-feng Kang from the Department of General Surgery, the First Affiliated Hospital of Medicine School, Shihezi University, Xinjiang, China.

\section{References}

1. Parkin DM, Bray F, Ferlay J and Pisani P: Global cancer statistics, 2002. CA Cancer J Clin 55: 74-108, 2005.

2. Sugano K: Screening of gastric cancer in Asia. Best Pract Res Clin Gastroenterol 29: 895-905, 2015.

3. Gessner R and Tauber R: Intestinal cell adhesion molecules. Liver-intestine cadherin. Ann N Y Acad Sci 915: 136-143, 2000.

4. Wendeler MW, Drenckhahn D, Gessner R and Baumgartner W: Intestinal LI-cadherin acts as a $\mathrm{Ca}^{2+}$-dependent adhesion switch. J Mol Biol 370: 220-230, 2007.

5. Ito R, Oue N, Yoshida K, Kunimitsu K, Nakayama H, Nakachi K and Yasui W: Clinicopathological significant and prognostic influence of cadherin-17 expression in gastric cancer. Virchows Archiv 447: 717-722, 2005.
6. Su MC, Yuan RH, Lin CY and Jeng YM: Cadherin-17 is a useful diagnostic marker for adenocarcinomas of the digestive system. Mod Pathol 21: 1379-1386, 2008.

7. Yu QF, Dong WG and Ren JL: Knockdown of Li-cadherin increases metastatic behaviors of LoVo cells. J Cancer Res Clin Oncol 136: 1641-1649, 2010.

8. Liu QS, Zhang J, Liu M and Dong WG: Lentiviral-mediated miRNA against liver-intestine cadherin suppresses tumor growth and invasiveness of human gastric cancer. Cancer Sci 101: 1807-1812, 2010.

9. Zhang J, Liu QS and Dong WG: Blockade of proliferation and migration of gastric cancer via targeting $\mathrm{CDH} 17$ with an artificial microRNA. Med Oncol 28: 494-501, 2011.

10. Hinoi T, Lucas PC, Kuick R, Hanash S, Cho KR and Fearon ER: CDX2 regulates liver intestine-cadherin expression in normal and malignant colon epithelium and intestinal metaplasia. Gastroenterology 123: 1565-1577, 2002.

11. Barros R, da Costa LT, Pinto-de-Sousa J, Duluc I, Freund JN, David L and Almeida R: CDX2 autoregulation in human intestinal metaplasia of the stomach: Impact on the stability of the phenotype. Gut 60: 290-298, 2011.

12. Ge J, Chen Z, Wu S, Yuan W, Hu B and Chen Z: A clinicopathological study on the expression of cadherin-17 and caudal-related homeobox transcription factor (CDX2) in human gastric carcinoma. Clin Oncol (R Coll Radiol) 20: 275-283, 2008.

13. GrötzingerC,Kneifel J,Patschan D, Schnoy N, Anagnostopoulos I, Faiss S, Tauber R, Wiedenmann B and Gessner R: LI-cadherin: A marker of gastric metaplasia and neoplasia. Gut 49: 73-81, 2001.

14. Ko S, Chu KM, Luk JM, Wong BW, Yuen ST, Leung SY and Wong J: CDX2 co-localizes with liver-intestine cadherin in intestinal metaplasia and adenocarcinoma of the stomach. J Pathol 205: 615-622, 2005.

15. Cui J, Chen Y, Chou WC, Sun L, Chen L, Suo J, Ni Z, Zhang M, Kong X, Hoffman LL, et al: An integrated transcriptomic and computational analysis for biomarker identification in gastric cancer. Nucleic Acids Res 39: 1197-1207, 2011.

16. Correa P: Human gastric carcinogenesis: A multistep and multifactorial process - First American Cancer Society Award Lecture on Cancer Epidemiology and Prevention. Cancer Res 52: 6735-6740, 1992.

17. Yasui W, Oue N, Sentani K, Sakamoto N and Motoshita J: Transcriptome dissection of gastric cancer: Identification of novel diagnostic and therapeutic targets from pathology specimens. Pathol Int 59: 121-136, 2009.

18. Ko S, Chu KM, Luk JM, Wong BW, Yuen ST, Leung SY and Wong J: Overexpression of LI-cadherin in gastric cancer is associated with lymph node metastasis. Biochem Biophys Res Commun 319: 562-568, 2004.

19. Wang J, Yu JC, Kang WM, Wang WZ, Liu YQ and Gu P: The predictive effect of cadherin-17 on lymph node micrometastasis in pN0 gastric cancer. Ann Surg Oncol 19: 1529-1534, 2012.

20. Xu Y, Zhang J, Liu QS and Dong WG: Knockdown of liver-intestine cadherin decreases BGC823 cell invasiveness and metastasis in vivo. World J Gastroenterol 18: 3129-3137, 2012.

21. Wu WK, Cho CH, Lee CW, Fan D, Wu K, Yu J and Sung JJ: Dysregulation of cellular signaling in gastric cancer. Cancer Lett 295: 144-153, 2010.

22. Song Y, Dong MM and Yang HF: Effects of RNA interference targeting four different genes on the growth and proliferation of nasopharyngeal carcinoma CNE-2Z cells. Cancer Gene Ther 18: 297-304, 2011.

23. Liu LX, Lee NP, Chan VW, Xue W, Zender L, Zhang C, Mao M, Dai H, Wang XL, Xu MZ, et al: Targeting cadherin-17 inactivates Wnt signaling and inhibits tumor growth in liver carcinoma. Hepatology 50: 1453-1463, 2009.

24. Kwak JM, Min BW, Lee JH, Choi JS, Lee SI, Park SS, Kim J, Um JW, Kim SH and Moon HY: The prognostic significance of E-cadherin and liver intestine-cadherin expression in colorectal cancer. Dis Colon Rectum 50: 1873-1880, 2007.

25. Takamura M, Ichida T, Matsuda Y, Kobayashi M, Yamagiwa S, Genda T, Shioji K, Hashimoto S, Nomoto M, Hatakeyama K, et al: Reduced expression of liver-intestine cadherin is associated with progression and lymph node metastasis of human colorectal carcinoma. Cancer Lett 212: 253-259, 2004.

26. Tait SW and Green DR: Mitochondria and cell death: Outer membrane permeabilization and beyond. Nat Rev Mol Cell Biol 11: 621-632, 2010. 